International Journal of Wireless \& Mobile Networks (IJWMN) Vol. 5, No. 1, February 2013

\title{
Energy Efficient Routing of Wireless Sensor Networks Using Virtual Backbone and life time Maximization of Nodes
}

\author{
Umesh B.N ${ }^{1}$, Dr G Vasanth ${ }^{2}$ and Dr Siddaraju ${ }^{3}$ \\ ${ }^{1}$ Research Scholar, ${ }^{2}$ Professor \& Head, Dept of Computer Science, Govt Engg College, \\ K.R Pate and ${ }^{3}$ Professor \& Head, Dept of Computer Science, Dr. AIT, Bangalore \\ ${ }^{1}$ umeshbn5@gmail.com, ${ }^{2}$ gvasanth_ss@yahoo.co.in, ${ }^{3}$ Siddaraju.ait@gmail.com
}

\begin{abstract}
Different approaches have been proposed based on routing in WSN (Wireless Sensor Nodes) for Energy Efficient routing in Multi hop Networks and some localized based on geographical routing schemes have been also proposed. The key concept in virtual backbone scheduling is to minimize the energy consumption and more throughputs. To achieve QOS and fault tolerance of these backbone nodes in Multi hop Networks requires stable links. Hence existing localized routing in virtual backbone scheduling cannot guarantee the energy efficient routes. In this paper we propose an energy efficient routing for Virtual Back Bone Nodes (VBS) in which it maximizes the node life and turns off it's radio when they are in sleep mode, in order to consume less energy. A concept of Restricted Back Bone Neighborhood Routing is proposed, which assures the efficient routing with minimum energy consumption of nodes and also implemented the critical transmission radius for Backbone nodes.
\end{abstract}

Index terms: WSN, VBS, QOS, Critical Transmission radius.

\section{INTRODUCTION}

A wireless sensor network is a collection of nodes organized into a cooperative network. Each node consists of processing capability (one or more microcontrollers, CPUs or DSP chips), may contain multiple types of memory (program, data, and flash memories), have a RF transceiver (usually a single omni-directional antenna), have a power source (e.g., batteries and solar cells), and accommodate various sensors and actuators. The nodes communicate wirelessly and often self-organize after being deployed in an ad hoc fashion. Systems of 1000s or even 10,000 nodes are anticipated. The unique feature of Wireless Sensor Network (WSN) is the co-operative feature of sensor nodes. Wireless Sensor Networks are different from traditional network because of their own design and resource constraints and having so many challenges and issues, among these all maximizing the network life by reducing power consumption is one of the most important challenges for WSN.

Micro Electro-Mechanical systems have made extensive product developments which are of low-power and low cost sensors and less cost, such that the sensors are widely deployed. These Sensor nodes are widely used for monitoring applications like whether monitoring, agriculture monitoring etc. Conservation of energy and scalability are the two most important issues in WSN because Nodes are powered with the battery. The WSN contains three parts: Data Collection, BS (Base Station) and Management centre. Hence there is no particular 
International Journal of Wireless \& Mobile Networks (IJWMN) Vol. 5, No. 1, February 2013

infrastructure. Flooding is a kind of broadcasting in sensor network. Energy is consumed when each node retransmits the broadcasted massage which it receives. When there is an interference, node raises its energy consumption for packet retransmission which utilizes more energy, this problem is called as Broadcast Storm Problem [15][16] Extensive research has been performed on formation of back bone nodes. These backbone nodes will eliminate unwanted transmission links by turning off the redundant nodes but still backbone node assures network connectivity to deliver the data efficiently.

Subset of active nodes are called as backbone, hence backbone network is to be connected. Backbone is mainly used to improving the routing procedure which increases bandwidth efficiency, decreases overall energy consumption and increases node lifetime. Among computational functions the radio consumes more energy [1]. Hence we focus on load distribution of nodes by creating the Backbone nodes which are active when any massage sending process takes place and goes to sleep by turning off their radios. Hence it does not disturb the communication quality. However creating a single backbone node does not provide the maximum life time of node; hence it is best idea to create multiple disjoint Connected Dominated Sets (CDS) which works efficiently and can adapt to network topology changes [2].CDS can be classified into different types 1) UDG ( Unit Disk Graph) and 2) DGB ( Disk Graphs with Bidirectional links. UDG and DGB is an NP-hard [14] [17]. Here non CDS nodes are put into sleep mode so as to conserve overall energy of the network [11]. Backbone nodes use the deterministic scheme to keep the backbone node small with high computation [12]. Thus this scheme guarantees the CDS in connected network [13]. In previous localized routing the consumption of energy was more than it's optimal. In this paper we study energy efficient routing using virtual back bone to maximize its life time. Contributions are as follows:

- The Network is divided into zones or areas, where each backbone node present in these zones or areas has transmission radius and is restricted to find the neighbor backbone node within their transmission radius so as to find the stable links.

- A technique called Restricted Back Bone Neighborhood routing is proposed, which assures the efficient routing with minimum energy consumption. In which the backbone nodes select the neighboring backbone node which is inside the transmission radius and forms the Connected Dominated Set (CDS) of backbones from the sink node. If no node found inside the transmission radius, the backbone node extends its transmission radius called as critical transmission radius to find the next backbone node and forms a Connected Dominated Set of backbone nodes.

- A Sink Node is placed at the center which is active all the time

- Back Bone Nodes can get their lifetime using duty cycle specified to the particular zone or area.

Thus the backbone reduces communication overhead, increases bandwidth efficiency, decreases overall network energy consumption and last increases the node life span in the WSN [14] 

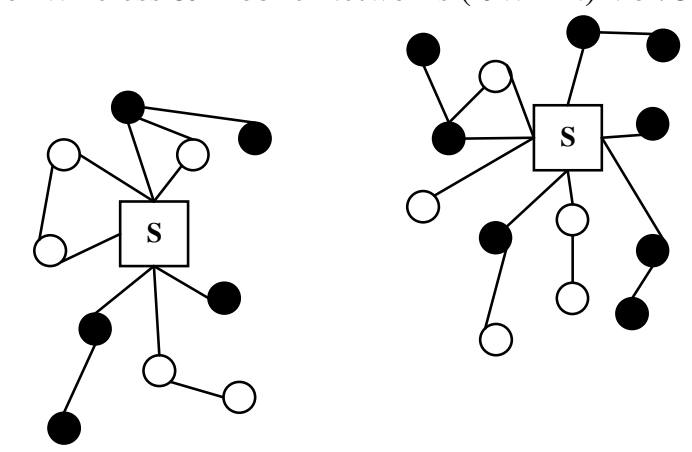

Backbone Nodes Connected from sink

Fig 1: Formation of CDS from Sink Node

The rest of the paper is organized as follows:

Section II presents Problem Definition, Section III presents Related Work, Section IV presents Implementation, Section V presents simulation results and Section VI presents Conclusion and Future work.

\section{Problem Definition}

The following problem is about Wireless Sensor Network (WSN) that sensor nodes are deployed randomly in a field. Thus a battery is the main energy source for the sensor nodes. For the whole network there is an only one sink node, which is active all time and has more energy back up. Sensor nodes have links which are of identical way i,e bidirectional communication range. The radio of sensor node is the one which consumes more energy resources. Many of the algorithms select backbone node without considering any geographical information. Hence we consider formation of stable backbone links and the scheduling of radio which was proposed for static nodes previously.

\section{RELATED WORK}

\section{A. Network Model and Problem Definition}

In Network Model, Let us consider a $\mathrm{V}$ as a set of $\mathrm{n}$ wireless devices which is widely distributed in a compact and convex region. Hence the region $\Omega$ is a unit-area square or a unitarea disk. Each node position is known by some low power receivers like GPS When singlehop broadcasting is done. For each node we can get the location information of all nodes within its transmission radius. The Transmission radius ' $r$ ' is set to all nodes uniformly. Whereas when considered about multi hop network modeled by a graph which is known as communication graph which is denoted as $G(V, r)$, where two nodes are connected in $G(V, r)$ if it is in Euclidean distance.

\section{B. Localized Routing}

In geometric area where multi-hop wireless networks allow the idea called localized routing protocol. The popular and widely localized protocol based on routing is greedy routing. The greedy routing is able to find the shortest paths between nodes by using local information, without global network topology. 
The routing in greedy takes place when a node forwards information to the nearest destination node. Greedy routing is simple and efficient but cannot guarantee the packet delivery, while face routing can guarantee the delivery but may take a lengthy exploration. One natural improvement is to combine greedy routing and face routing by using face routing to recover the route after greedy method fails in local minimum. Many routing protocols [3], [4], [5], [6] used this approach, such as greedy face routing (GFG) [4].

Stojmenovis proposed power-aware localized routing which is based on the combination of remaining battery power in nodes and based on transmission power related to node distance [3]

Seade et al [18] proposed a power-aware greedy routing which concentrates on transmission power and also the reliability of each link. This focuses on routing without lossy links. Hence showed the significant enhancement in delivery rate and energy efficiency in lossy network

\section{Energy Efficient Routing}

Since energy is a scarce resource which limits the life of wireless networks, a number of energy efficient routing protocols [7], [8], [3], [4], [5], [6], [9], [10] have been proposed recently using a variety of techniques. Classical routing algorithm maybe adapted to take into account energyrelated criteria rather than classical metrics such as delay or hop distance. Most of the proposed energy-aware metrics are defined as a function of the energy required to communicate on a link [3] or a function of the nodes remaining lifetime [3]. However, to minimize the global consumed energy of selected route, most of minimum energy routing algorithms are centralized algorithms. In this paper, we focus on stateless localized routing. Thus, we only review the following related work about energy efficient techniques for localized routing which address how to save energy when making local routing decision.

\section{IMPLEMENTATION}

In this section we describe about the backbone scheduling to find the efficient routing based on localized efficient back bone routing. The complete modules have been discussed below.

\section{A. Schedule Transition Graph (STG)}

STG is shown in the below figure, it is a centralize approximation algorithm. The time scale is shown in horizontal which is counted in rounds. The possible states of the backbone nodes are vertically listed in each round. The number of backbone is equal to the number of possible states in each round. There is an one to one mapping between state and backbone. Energy is used in 1 round which represents the time laps during each round when consumes energy. Transition of node is not allowed when there is state depletion in node. 


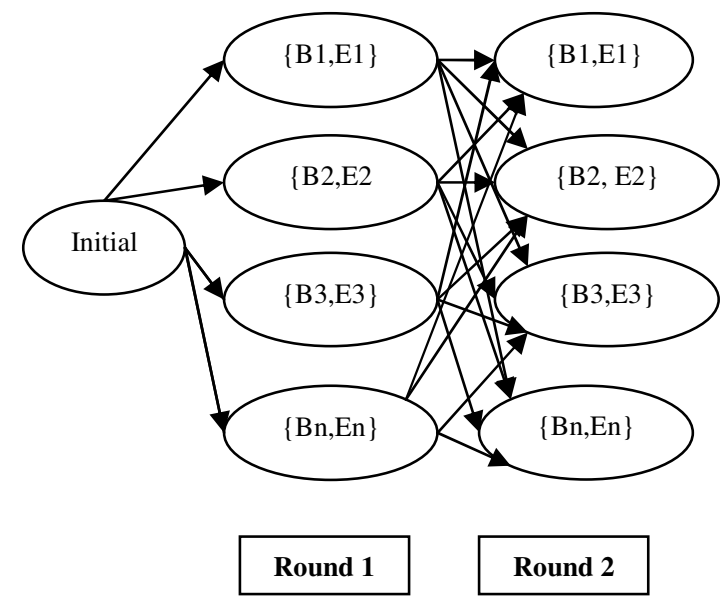

Fig 2: STG Scheduling.

Where B is a set of backbone nodes. Backbone nodes work with rounds. Connectivity of all is a network connected sub-graph and all other nodes are 1-hop away from node in $\mathrm{B}_{\mathrm{i}}$.

\section{B. Localized Energy Efficient Routing}

This section describes the details of our routing concept based on localized routing. This is a kind of greedy method routing. In greedy routing the node selects the next hop neighbor which is based on its distance to destination. Hence such routing may not be energy efficient. So as to achieve energy efficient, the forwarding link should have larger energy mileage. To consume less energy the distance traveled totally should be small. Thus a new concept called restricted region is introduced to restrict forwarding directions.

- The backbone node with a message which has to be forwarded finds the neighbor backbone node among the neighbors present inside the restricted area. The best neighbor has the maximum energy mileage or a stable link

- If no backbone node is present in the restricted area, it extends its critical transmission radius and finds the best node.

- If in case no node present in the area or zone then the classic greedy routing takes place.

The dataflow of this process is shown in Fig 3.

\section{Critical Transmission Radius}

In this study, while routing the packets from source to destination, the intermediate nodes may drop the packets before it reaches the destination, this happen because it is not able to find the better neighbor node. Hence to ensure successful and efficient routing, the backbone node should have sufficiently large transmission radius so as to find the better neighbor backbone node. 
The critical transmission radius is given as

$$
\rho_{A}\left(P_{n}\right)=\sqrt{\frac{\beta_{A} \ln (D \cdot n)}{n \pi}}
$$

$\rho_{A}$ is an generalized routing method and $P_{n}$ is number of nodes in network.

$\beta_{A}$ is ratio of center of intermediate node $u$ with radius $r$ of the forwarding area from where the intermediate backbone node $u$ can choose its next neighbor backbone node $w$

\section{PERFORMANCE EVALUATION}

In this section we describe our simulation and Methodology as well comparing performance through simulation results of Energy model, Network lifetime of backbone nodes and Efficient Routing.

\section{A. Simulation Methodologies}

To find out the efficient routing of backbone node and network life time we simulate our proposed system using ns 2 simulator.

\section{B. Simulation Configurations}

Our simulation is conducted with the Network Simulator (NS) 2.34 environment on a platform with GCC-4.3 and Ubuntu 11.10. The system is running on a laptop with Core 2 Duo T7250 CPU and 3GB RAM. In order to better compare our simulation results with other research works, we adopted the default scenario settings in NS 2.35. 
International Journal of Wireless \& Mobile Networks (IJWMN) Vol. 5, No. 1, February 2013

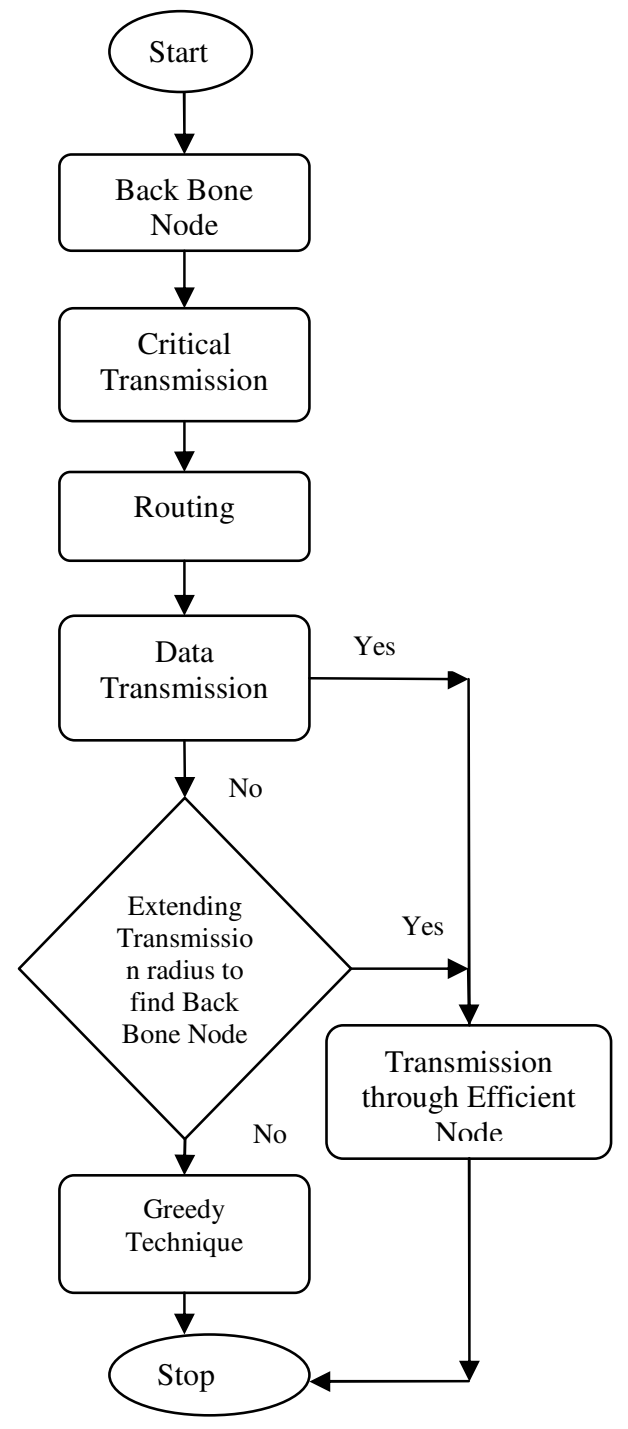

Fig 3: Data Flow Diagram

\section{Network Life Time of Back Bone nodes}

Here in this we present the result of the proposed technique. The result is shown on ns2 simulator, the concept of VBS and localized routing produces the best result in finding out the stable back bone node links which are area restricted. This is shown in Fig 8

\section{Energy Model.}

Energy is used only when there is a massage transfer, and turns off its radio, when a node goes to sleep mode. Energy is mainly used only when there is a message transfer which should satisfy the QOS. Consumption of less energy is shown in Fig 6 
International Journal of Wireless \& Mobile Networks (IJWMN) Vol. 5, No. 1, February 2013

\section{E .Efficient Routing}

In this Results we represent the efficient routing, which is formed by CDS by creating the stable links between the back bone nodes. Routing efficiency is shown in Fig 7

Table 1 shows the parameter used to simulate the proposed system

\begin{tabular}{|c|l|l|}
\hline SI No & Parameter & Value \\
\hline 1 & Number of Nodes & 35 \\
\hline 2 & Topology Dimension & $670 \mathrm{~m}$ x 670m \\
\hline 3 & Traffic Type & CBR \\
\hline 4 & Radio Propagation Model & Two-Ray Ground \\
\hline 5 & MAC Type & 802.11 MAC Layer \\
\hline 6 & Packet Size & 512 Bytes \\
\hline 7 & Antenna Type & Omni Dimension \\
\hline
\end{tabular}

Table 1: Parameters Used

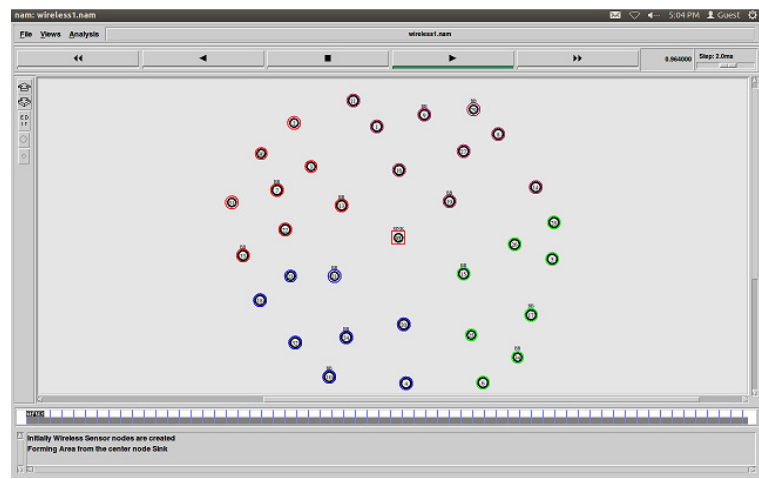

Fig 4: Initial WSN Setup with Backbone Nodes

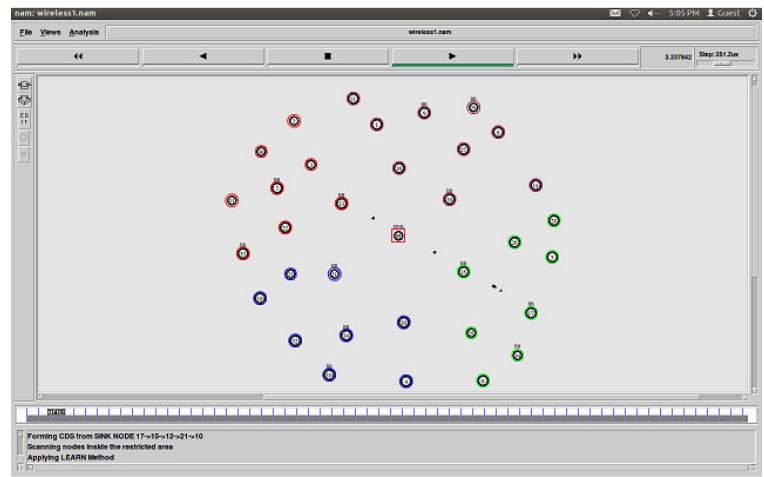

Fig 5: Routing through Backbone Nodes 


\section{SIMULATION AND ANALYSIS}

\section{Energy Consumption}

In WSN Energy is the major concern, hence to minimize energy consumption and for more throughput, creating virtual nodes is the idea. It is reasonable to approximatively calculate the energy consumed according to the data transmission Fig 6 shows the energy consumption of back bone nodes during the transmission of data and while in sleep mode it turns off the radios for saving energy. The graph is plotted for the no of backbone nodes present in the network and their energy consumption. Therefore the energy consumption of the proposed scheme is not higher than that of existing scheme. The blue line shows the energy consumed by the backbone nodes in the overall WSN

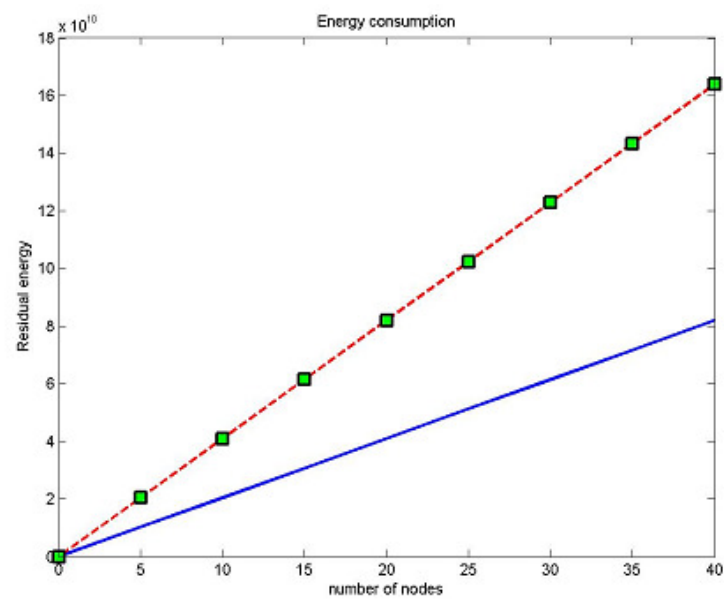

Fig 6: Energy Consumption

\section{Efficient Routing}

In efficient routing the total length of the path found by LEARN is within a constant optimum. The proposed system is compared with existing localized routing methods and proves that it can guarantee the energy efficient routes from source to destination. Extensive simulation is conducted to study the performance of the LEARN Routing. This shows that LEARN Localized routing method guarantees the energy efficient routes in random network with high probability. Fig 7 shows the backbone nodes with efficient routing. The simulation results for throughput is shown as efficient routing 
International Journal of Wireless \& Mobile Networks (IJWMN) Vol. 5, No. 1, February 2013

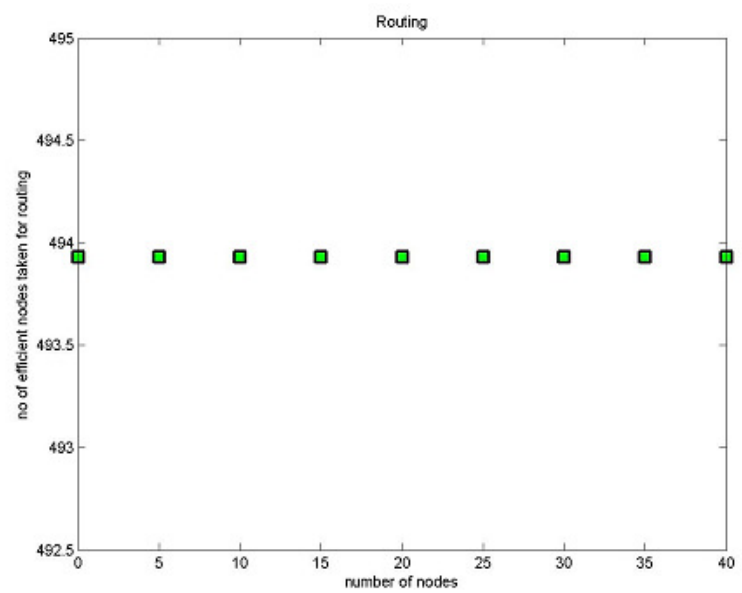

Fig 7: Efficient Routing

\section{Node Lifetime}

In node lifetime backbone nodes achieves the prolong network life. Identical initial energy and the imbalanced energies are used as energy configurations. Initially the transmission range is fixed, later based on the critical transmission the transmission radius can be extended. Fig 8 shows the achieved network lifetime. By distributing the energy uniformly. The blue line indicates the network lifetime of backbone nodes

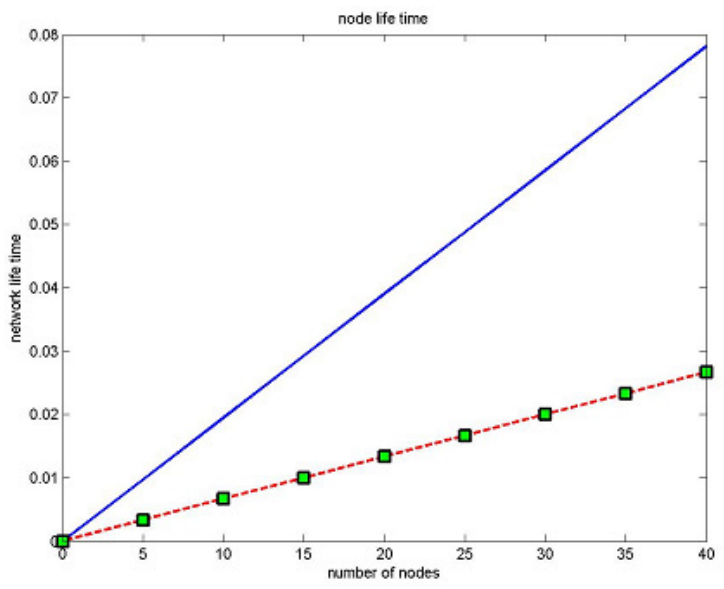

Fig 8: Node Lifetime

\section{Delay}

This simulation results shows that there is a less delay in finding out efficient path and constructing CDS from the sink node it is shown in Fig 9 
International Journal of Wireless \& Mobile Networks (IJWMN) Vol. 5, No. 1, February 2013

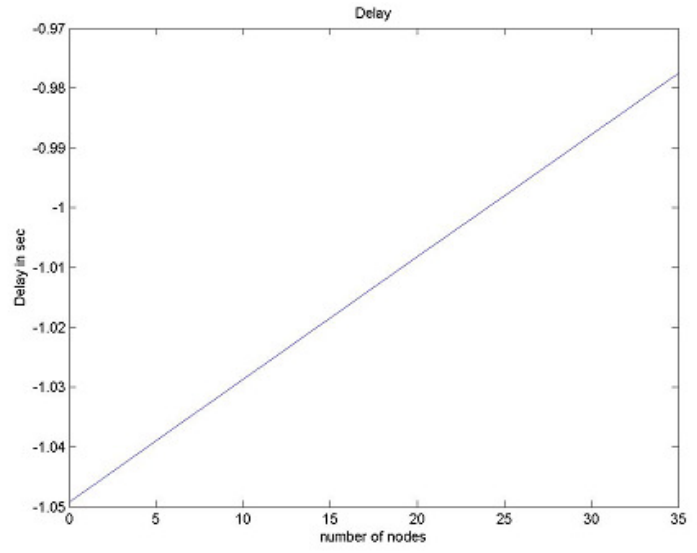

Fig 9: Delay

\section{Conclusion}

Energy Efficient communication is needed for WSN so as to work for a longer period because of battery powered, therefore network lifetime through power aware node organization is high desirable. An efficient method based on low energy consumption scheduling has to be implemented so as to schedule the node activity between sleep and active state. One solution is to create a backbone node and make their radios off when they are in sleep mode and the routing should be energy efficient by making the network life time prolong.

\section{REFERENCES}

[1] Shnayder, M. Hempstead, B.-r. Chen, G. W. Allen, and M. Welsh, "Simulating the power consumption of large-scale sensor network applications," in Proc. of SenSys '04. New York, NY, USA: ACM, 2004, pp. 188-200.

[2] C. Misra, R. Mandal, "Rotation of cds via connected domatic partition in ad hoc sensor networks," IEEE Trans. on Mobile Computing, pp. 488-499, 2009.

[3] I. Stojmenovic and X. Lin, "Loop-Free Hybrid Single-Path/ Flooding Routing Algorithms with Guaranteed Delivery for Wireless Networks," IEEE Trans. Parallel and Distributed Systems, vol. 12, no. 10, pp. 1023-1032, Oct. 2001.

[4] P. Bose, P. Morin, I. Stojmenovic, and J. Urrutia, "Routing with Guaranteed Delivery in Ad Hoc Wireless Networks,” ACM/ Kluwer Wireless Networks, vol. 7, no. 6, pp. 609-616, 2001.

[5] F. Kuhn, R. Wattenhofer, Y. Zhang, and A. Zollinger, "Geometric Ad-Hoc Routing: Of Theory and Practice,” Proc. ACM Symp. Principles of Distributed Computing (PODC), 2003.

[6] F. Kuhn, R. Wattenhofer, and A. Zollinger, "Worst-Case Optimal and Average-Case Efficient Geometric Adhoc Routing, ," Proc. ACM Int'l Symp. Mobile Ad Hoc Networking and Computing (MobiHoc), 2003.

[7] K. Kar, M. Kodialam, T. Lakshman, and L. Tassiulas, "Routing for Network Capacity Maximization in Energy-Constrained Ad-Hoc Networks,” Proc. IEEE INFOCOM, 2003.

[8] J.-H. Chang and L. Tassiulas, "Energy Conserving Routing in Wireless Ad-Hoc Networks," Proc. IEEE INFOCOM, 2000. 
International Journal of Wireless \& Mobile Networks (IJWMN) Vol. 5, No. 1, February 2013

[9] Q. Li, J. Aslam, and D. Rus, "Online Power-Aware Routing in Wireless Ad-Hoc Networks," Proc. ACM Mobicom, 2001.

[10] J. Kuruvila, A. Nayak, and I. Stojmenovic, "Progress and Location Based Localized Power Aware Routing for Ad Hoc and Sensor Wireless Networks," Int'1 J. Distributed Sensor Networks, vol. 2, pp. 147-159, 2006.

[11] B. Chen, K. Jamieson, H. Balakrishnan, and R. Morris, "SPAN: An energy-efficient coordination algorithm for topology maintenance in ad hoc wireless networks," Wireless Networks, ACM, vol. 8, no. 5, pp. 481-494, 2002.

[12] F. Dai and J. Wu, "On constructing k-connected k-dominating set in wireless ad hoc and sensor networks," Journal of Parallel Distributed Computing (JPDC), Elsevier, vol. 66, no. 7, pp. 947-958, 2006.

[13] An extended localized algorithm for connected dominating set formation in ad hoc wireless networks," IEEE Transactions on Parallel Distributed Systems, vol. 15, no. 10, pp. 908-920, 2004.

[14] M. Rai, Sh. Verma, and Sh. Tapaswi, "A Power Aware Minimum Connected Dominating Set for Wireless Sensor Networks," Journal of networks, Vol. 4, no. 6, August 2009.

[15] J. Akbari Torkestani, M. R. Meybodi, "An intelligent backbone formation algorithm for wireless ad networks based on distributed learning automata," Computer Networks 54, pp. 826-843, 2010.

[16] S. Hussain, M. I. Shafique, and L. T. Yang, "Constructing a CDS-Based Network Backbone for Energy Efficiency in Industrial Wireless Sensor Network," In Proceedings of HPCC, pp. 322-328, 2010.

[17] Z. Liu, B. Wang, and Q. Tang, "Approximation Two Independent Sets Based Connected Dominating Set Construction Algorithm for Wireless Sensor Networks," Inform. Technol. J., Vol. 9, Issue 5, pp. 864-876, 2010.

[18] K. Seada, M. Zuniga, A. Helmy, and B. Krishnamachari, "Energy- Efficient Forwarding Strategies for Geographic Routing in Lossy Wireless Sensor Networks," Proc. ACM Int'l Conf. Embedded Networked Sensor Systems (Sensys), 2004. 